\title{
Transition From an ICU Ventilator to a Portable Home Ventilator in Children
}

\author{
L Denise Willis, Gary Lowe, Phyllis Pearce, Beverly J Spray, Randy Willis, Angela Scott, \\ John L Carroll, and Amit Agarwal
}

\begin{abstract}
BACKGROUND: The transition from an ICU ventilator to a portable home ventilator (PHV) for children requiring long-term mechanical ventilation is a crucial step in preparing for discharge home and may not be successful on the first attempt. A review of this process at our institution revealed that some children required multiple trials before they were able to tolerate a PHV. A protocol was developed to standardize the transition process and reduce the number of failed attempts. Key features of the protocol included a transition readiness assessment and criteria for changing to the PHV. METHODS: A retrospective chart review was completed to evaluate the process of changing to a PHV before and after the protocol was in place during the time period of 2011-2018. Primary outcome measures included the number of transition attempts and the length of time to achieve successful transition. A successful transition attempt was defined as the ability to tolerate a PHV for $14 \mathrm{~d}$. RESULTS: The study included 56 children $\leq 3 \mathrm{y}$ old with a tracheostomy who required long-term ventilator support. The majority of subjects were from the neonatal ICU and had a diagnosis of bronchopulmonary dysplasia. There was a significant decrease in the number of attempts $(P=.005)$ and in the length of time $(P=.01)$ to successfully transition to a PHV for those who underwent the protocol. CONCLUSIONS: The process of changing from an ICU ventilator to a PHV in children requiring long-term mechanical ventilation was improved through the use of a standardized protocol. Both the number of failed attempts and the length of time to achieve successful transition were reduced when the protocol was applied. Further study is needed to evaluate other medical and nonmedical factors that may affect successful transition to a PHV. Key words: ventilator-dependent children; long-term mechanical ventilation; portable home ventilator; pediatric ventilation; home mechanical ventilation; pediatric tracheostomy; transition to home ventilator; bronchopulmonary dysplasia; chronic respiratory failure; discharge planning. [Respir Care 2020;65(12):1791-1799. (C) 2020 Daedalus Enterprises]
\end{abstract}

\section{Introduction}

Children requiring long-term mechanical ventilation represent a diverse, complex, and medically fragile group that continues to expand in number due to advances in medicine

Ms Willis and Mr Willis are affiliated with Respiratory Care Services, Arkansas Children's Hospital, Little Rock, Arkansas. At the time of this research, Mr Lowe (now retired) was affiliated with Respiratory Care Services, Arkansas Children's Hospital, Little Rock, Arkansas. Ms Scott is affiliated with the Department of Nursing, Arkansas Children's Hospital, Little Rock, Arkansas. Ms Pearce and Dr Spray are affiliated with Arkansas Children's Research Institute, Little Rock, Arkansas. Drs Carroll and Agarwal are affiliated with the Department of Pediatrics, University of Arkansas for Medical Sciences, Little Rock, Arkansas. and technology. ${ }^{1}$ It has been well established that these children thrive outside of the hospital setting with improvements in both physical and psycho-social well-being. ${ }^{2}$ The cost for supporting mechanical ventilation in the home is typically less than in the hospital setting. ${ }^{3-5}$ The estimated prevalence of children $\leq 18$ y old utilizing home

\footnotetext{
Ms Willis presented a version of this paper at the AARC Congress 2018, held December 4-7, 2018, in Las Vegas, Nevada.

The authors have disclosed no conflicts of interests.

Correspondence: Amit Agarwal MD. E-mail: AgarwalAmit@uams.edu.
}

DOI: $10.4187 /$ respcare.07641 
mechanical ventilation in the Unites States is 4.7-6.4 children per $100,000 .{ }^{6}$ Although chronic respiratory failure represents the primary need for prolonged ventilation,

\section{SeE the Related Editorial on Page 1933}

underlying diagnoses vary and may include bronchopulmonary dysplasia (BPD), neuromuscular disease, congenital heart disease, spinal cord injury, traumatic brain injury, and many more. This population is relatively small compared to the total number of children with special health care needs, but it is one of the highest-cost and resource-utilization groups of children. ${ }^{7}$

In-patient stays can be lengthy following tracheostomy placement for long-term ventilator support. ${ }^{8,9}$ Length of stay (LOS) can be influenced by both medical and nonmedical factors, such as clinical stability, disease complexity, progress of caregiver training, availability of home nursing, and social issues. ${ }^{4,10-13}$ Caregiver training and discharge planning are crucial steps that must be completed before the child requiring long-term ventilation can be safely released from the hospital. Use of a standardized process for discharge at one pediatric institution was associated with a reduced LOS for ventilator-dependent children. ${ }^{14}$

The transition from an ICU ventilator to a portable home ventilator (PHV) is an important consideration in the discharge planning process that may not always be successful on the first attempt. There are differences between commercially available portable home ventilators because ventilator modes, settings terminology, and other options are generally not similar and PHVs have minimum patient weight requirements. ${ }^{15}$ These differences may not have a major impact in older children and adults, but they could affect infants and younger children to a greater degree. While some guidance exists for weaning from ventilator support in the hospital setting, to our knowledge there are no evidence-based guidelines that provide recommendations for best practice in changing from an ICU ventilator to a PHV for children requiring long-term mechanical ventilation. ${ }^{16}$ Although this step has been identified as part of the discharge process, the topic has received little attention. ${ }^{12}$ The few clinical practice guidelines available regarding pediatric chronic ventilation do not specifically address this subject. ${ }^{17-19}$

A review of the transition process from an ICU ventilator to a PHV at our institution revealed that some children experienced multiple unsuccessful trials before they were able to tolerate the PHV. There was no uniform approach in this process, and the approach tended to vary depending on the attending physician. As part of a quality-improvement initiative, a protocol was developed in an effort to standardize the transition process from an ICU ventilator to a PHV and to reduce the number of failed transition attempts, which may have an impact on hospital LOS.

\section{QUICK LOOK}

\section{Current knowledge}

Consensus guidelines are lacking for the transition from an ICU ventilator to a portable home ventilator (PHV) for children who require long-term mechanical ventilation. This process may not be successful on the initial attempt and may require return to the ICU ventilator when not tolerated. The inability to tolerate a PHV could contribute to prolonged length of stay.

\section{What this paper contributes to our knowledge}

A protocol with a standardized approach for the process of changing from an ICU ventilator to a PHV that includes consideration of underlying medical conditions, other system comorbidities, narcotic dependence, and optimization of the PHV's secondary settings to improve patient-ventilator synchrony may decrease the number of failed attempts and reduce the time to successful transition. Achieving successful transition to a PHV earlier could allow for sooner discharge home. Total length of stay may be an inaccurate measure of successful transition due to medical and nonmedical factors.

The majority of children who require long-term mechanical ventilation at our institution are transferred from an ICU to an intermediate care area for stable children receiving long-term invasive ventilation. This unit has a designated area for ventilator-dependent children $<3$ y of age, which essentially serves as a step-down unit from ICU. Attempts to transition to a PHV most commonly occur after transfer out of the ICU, but such transitions occasionally take place before moving to the intermediate care area. Typically, those that occur prior to transfer are a result of waiting for bed space availability. The focus of the protocol was the younger population that transfers to the non-ICU ventilator unit because there are more children in this age range who require long-term ventilator support at our institution. We hypothesized that utilizing a standardized protocol to manage the process for transition to a PHV would result in a lower number of failed attempts with the PHV.

\section{Methods}

This quality improvement project was deemed not human subject research by the local institutional review board. A retrospective chart review was completed at Arkansas Children's Hospital in Little Rock, Arkansas, to evaluate the process of changing to a PHV before and after the protocol was in place. Inclusion criteria were 


\section{Transition From ICU Ventilator to Home Ventilator}

Table 1. Readiness Criteria and Considerations for Transition to PHV

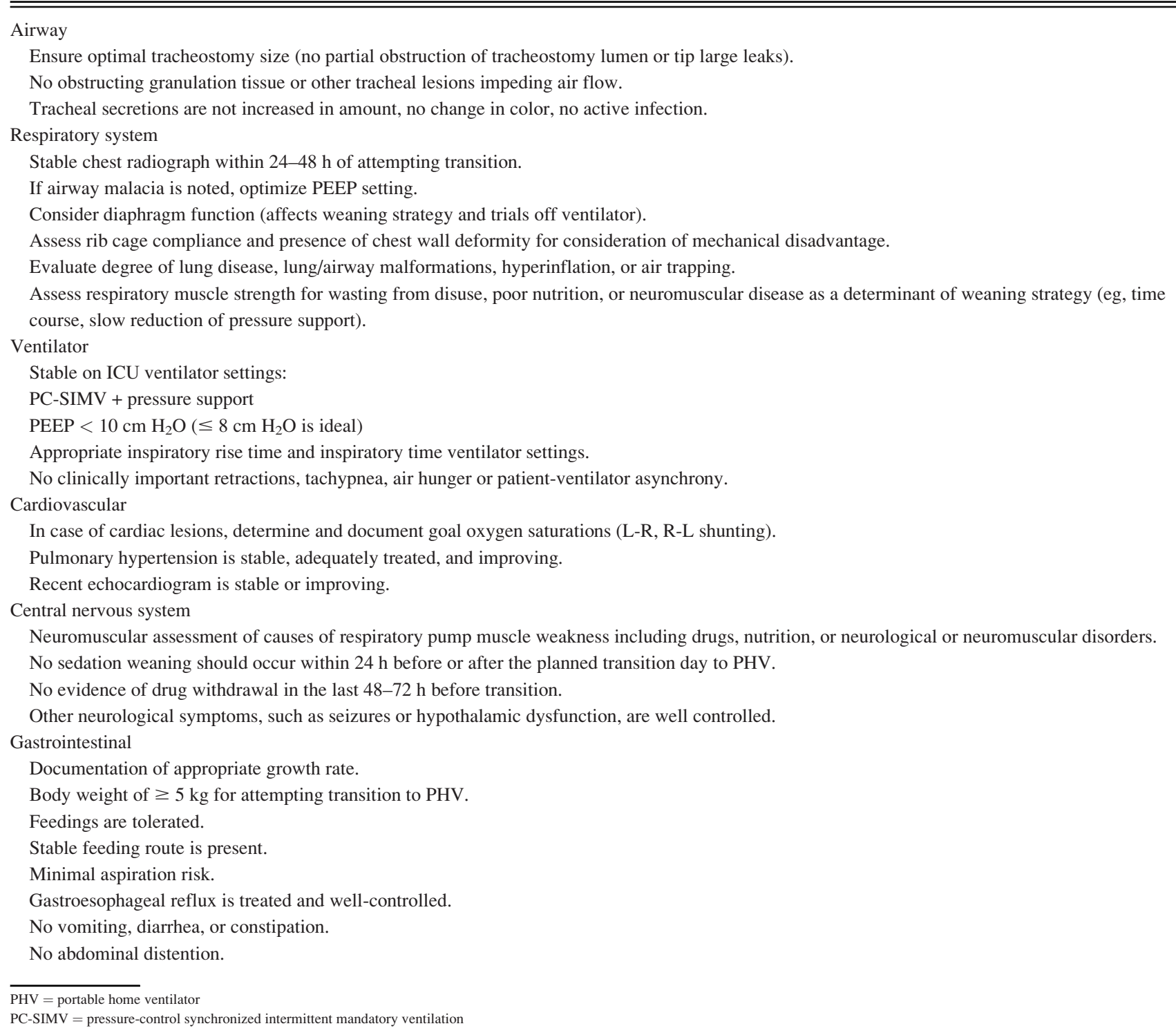

hospitalized children who required tracheostomy placement for long-term ventilator support and transferred from an ICU to the intermediate care unit during 2011-2018. Longterm ventilation was defined as the inability to wean from mechanical ventilation 3 months after initiation of ventilator support. ${ }^{20,21}$ Children were excluded if they died prior to discharge, were able to wean from the ICU ventilator and did not require a $\mathrm{PHV}$, or were $>3 \mathrm{y}$ old and transferred to other non-ICU ventilator areas.

The protocol incorporates a stepwise approach for transition to a PHV (TPHV) that includes a predetermined plan and strategy, which is established by an assigned primary pulmonologist. Key features of the protocol include standardized criteria for transition to a PHV, an assessment of readiness to transition to a $\mathrm{PHV}$, ventilator mode and settings recommendations, heightened observation and monitoring, and documentation during the transition attempt. The attending pulmonologist was responsible for execution and compliance with the protocol.

The readiness assessment is the first step of the protocol to ensure that the child is stable with current ICU ventilator settings and all potential system factors have been addressed before the PHV is attempted. For example, the child should have a stable chest radiograph, no evidence of sedation withdrawal symptoms, and adequate growth and nutrition. Table 1 includes the specific details of the readiness assessment. Once readiness to transition has been established, the protocol provides guidance for the process 


\section{Transition From ICU Ventilator to Home Ventilator}

Table 2. Transition to PHV Process*

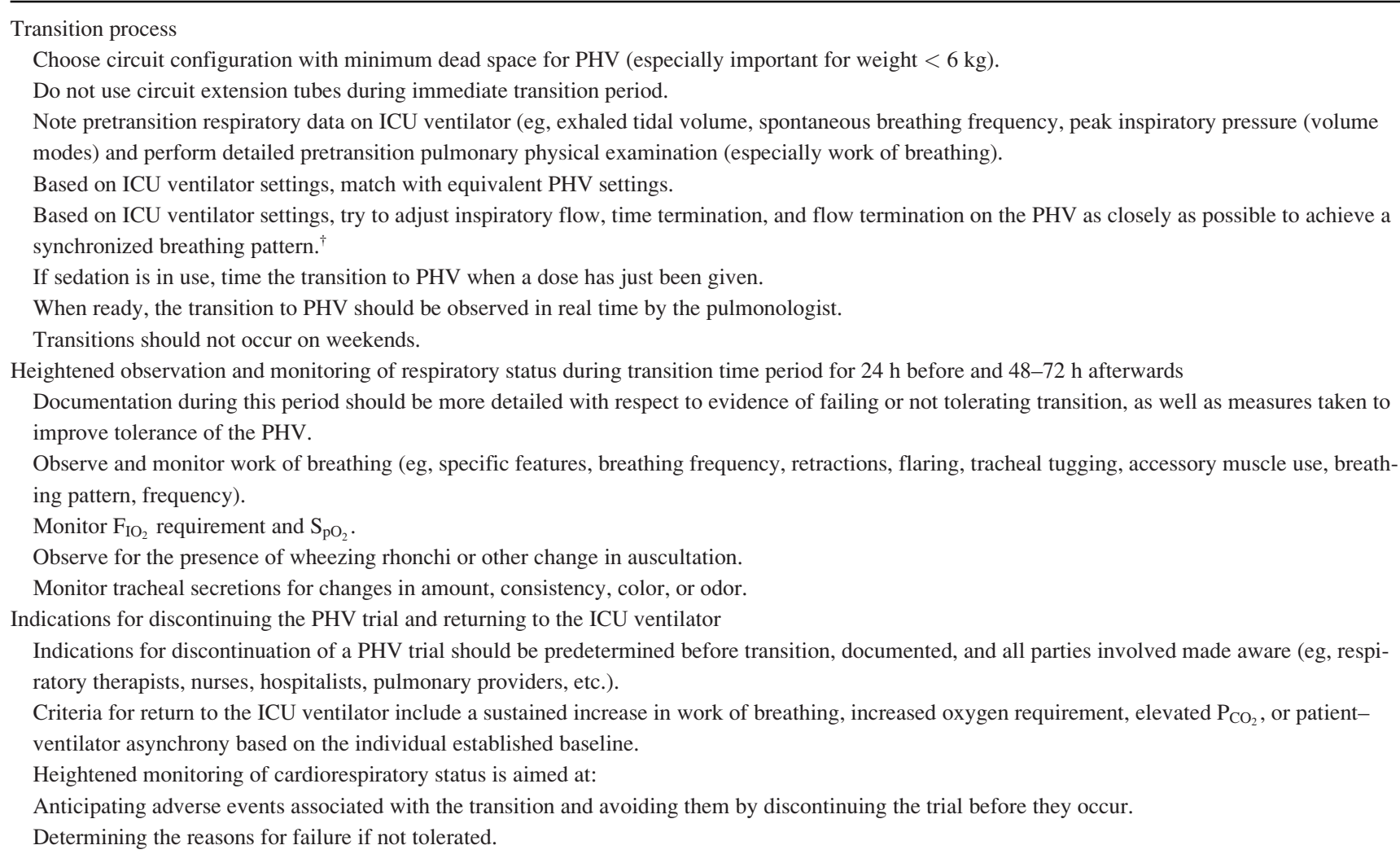

\footnotetext{
* When all of the readiness conditions are met.

${ }^{\dagger}$ PHV secondary settings for trigger, limit, and cycle variables.

$\mathrm{PHV}=$ portable home ventilator
}

of changing to a PHV. Prior to the transition attempt, minimize PHV circuit dead space, attempt to match equivalent PHV settings based on the ICU ventilator settings (including secondary settings such as trigger, limit, and cycle variables), and monitor the baseline work of breathing including spontaneous breathing frequency, exhaled tidal volume, and peak inspiratory pressure. Heightened monitoring and more detailed documentation should occur $24 \mathrm{~h}$ before and 48-72 $\mathrm{h}$ after a transition attempt to ensure readiness and to observe any signs of intolerance of the PHV. Criteria for discontinuing a PHV trial and returning to the ICU ventilator are predetermined and include a sustained increase in work of breathing, increased oxygen requirement, elevated $\mathrm{P}_{\mathrm{CO}_{2}}$, and patient-ventilator asynchrony. These criteria are modified and adjusted for each patient and established by baseline values. See Table 2 for additional details regarding steps for the transition process.

Children meeting inclusion criteria were divided into 2 groups: pre-TPHV protocol and post-TPHV protocol. The pre-TPHV group included subjects who were transitioned to a PHV during the years of 2011-2014 before the protocol was established. The time period of 2015-2018 comprised the post-TPHV protocol group. Collected data included age, weight, diagnosis, ICU location, number of transition attempts, number of failed attempts, length of time to achieve successful transition, and LOS. Disease severity between the pre-TPHV and post-TPHV groups was assessed by evaluating the continued need for ventilator support at $1 \mathrm{y}$ following tracheostomy placement and age at the time of discharge. The primary outcome was the number of failed attempts with a PHV, and the main secondary outcome was the length of time from the initial attempt to successful transition to a PHV. Other secondary outcomes were the total number of attempts and LOS. A subanalysis of the same outcomes was also completed for subjects from the neonatal ICU, those from other units, and those with a diagnosis of BPD for both pre-TPHV and post-TPHV groups. Weight at the time of successful transition was an additional outcome measure for subjects from the neonatal ICU and those with BPD. LOS in the neonatal ICU was also assessed and compared for subjects from the neonatal ICU.

A successful attempt was defined as demonstrating stability while receiving support with the PHV for at least 14 


\section{Transition From ICU Ventilator to Home Ventilator}

d without signs and symptoms of increased work of breathing, patient-ventilator asynchrony, or escalation of care requiring a return to the ICU ventilator. ${ }^{14,15}$ A return to the ICU ventilator after demonstrating clinical stability was not considered a failed attempt when the reason for return was acute illness (eg, enterovirus, rhinovirus) or following a scheduled procedure (eg, cardiac catheterization). These attempts were excluded and were not counted as failures. Acute illness was verified through clinical evaluation and laboratory evidence of concurrent illness such as respiratory pathogen panel or culture of tracheal secretions. The length of time to achieve a successful transition to PHV was determined from the date of the initial PHV attempt to the date of the successful attempt. Excluded attempts were not considered when calculating the number of days to successful transition to a PHV.

Outcome variables were compared before and after initiation of the protocol using independent sample $t$ tests for both the overall groups as well as subgroups. Data were screened for assumptions of normality and equal variance. For each outcome variable, these assumptions were met. Continuous variables were expressed as mean $\pm \mathrm{SD}$. Significance was considered at the $P<.05$, and all tests were 2-sided. Analyses were conducted using the Statistical Analysis Software 9.4 (SAS Institute, Cary, North Carolina).

\section{Results}

The chart review included 56 subjects who received a tracheostomy for long-term mechanical ventilation. There were 28 children in each group; however, one subject was excluded due to an atypical course with the PHV, leaving 27 children in the pre-TPHV protocol group. The most common diagnosis associated with the need for long-term ventilation was BPD. The majority of all subjects, $44 \%(n=14)$ had a diagnosis of $\mathrm{BPD}$, which included $37 \%(n=10)$ in the preTPHV protocol group and 50\% $(n=14)$ in the post-TPHV protocol group. Additional diagnoses related to the need for long-term ventilator support are included in Table 3. Males accounted for $56 \%(n=31)$ of all subjects in both groups, with $52 \%(n=14)$ and $61 \%(n=17)$ in the pre- and postTPHV protocol groups, respectively. There was no significant difference in gender $(P=.51)$ or mean age $(P=.78)$ at the time of the initial PHV attempt between subjects in the before and after groups. Fifty-six percent $(n=31)$ of all subjects were from the neonatal ICU, with $52 \%(n=14)$ in the pre-TPHV protocol group and $61 \%(n=17)$ in the postTPHV protocol group. Of the $44 \%(n=24)$ of subjects from other units, 35\% $(n=19)$ were from the pediatric ICU, with $33 \%(n=9)$ in the pre-TPHV protocol group and $36 \%(n=$ $10)$ in the post-TPHV protocol group; $9 \%(n=5)$ of subjects were from the cardiovascular ICU, with $15 \%(n=4)$ in the
Table 3. Primary Indication for Long-Term Ventilation

\begin{tabular}{|c|c|c|}
\hline & $\begin{array}{l}\text { Pre- } \\
\text { TPHV, } \\
n\end{array}$ & $\begin{array}{l}\text { Post- } \\
\text { TPHV, } \\
n\end{array}$ \\
\hline Bronchopulmonary dysplasia & 10 & 14 \\
\hline Airway malacia secondary to congenital heart disease & 5 & 2 \\
\hline Other airway malacia & 3 & 2 \\
\hline Pulmonary interstitial glycogenosis & 2 & 0 \\
\hline Hypoxic ischemic encephalopathy & 0 & 2 \\
\hline Other & 7 & 7 \\
\hline Congenital central hypoventilation syndrome* & & \\
\hline $\begin{array}{l}\text { Pulmonary hypoplasia secondary to congenital } \\
\text { diaphragmatic hernia* }\end{array}$ & & \\
\hline Diaphragm paralysis* & & \\
\hline Omphalocele* & & \\
\hline Posterior fossa mass status post ependymoma & & \\
\hline resection* & & \\
\hline Skeletal dysplasia* & & \\
\hline Spinal cord injury* & & \\
\hline Sequalae of acute respiratory distress syndrome $\dagger$ & & \\
\hline Congenital lobar emphysema $\dagger$ & & \\
\hline Congenital pulmonary airway malformation $\dagger$ & & \\
\hline Crouzon-like syndrome $\dagger$ & & \\
\hline Guillian-Barré syndrome with incomplete recovery $\dagger$ & & \\
\hline Hypotonia with myopathy $\dagger$ & & \\
\hline Upper-airway obstruction $\dagger$ & & \\
\hline $\begin{array}{l}\text { Pre-TPHV, } n=27 \text { subjects; Post-TPHV, } n=28 \text { subjects. } \\
\text { * Pre-TPHV. } \\
{ }^{+} \text {Post-TPHV. } \\
\text { TPHV }=\text { transition to portable home ventilator }\end{array}$ & & \\
\hline
\end{tabular}

pre-TPHV protocol group and $4 \%(n=1)$ in the post-TPHV protocol group.

Although the pathophysiology leading to the need for chronic ventilation was variable, subjects from both groups had comparable disease severity as the need for continued ventilator support at $1 \mathrm{y}$ post tracheostomy placement was similar. In the pre-TPHV group, $89 \%$ of subjects (24 of 27) remained ventilator-dependent 1 y after tracheostomy placement. Two of the subjects had weaned off the ventilator prior to discharge, and the status of one other was unknown due to transfer to an outof-state long-term care facility. In the post-TPHV group, $86 \%$ of subjects ( 24 of 28 ) remained ventilator-dependent 1 y after tracheostomy placement. One subject was weaned from ventilator support prior to discharge, and the status of 3 subjects at $1 \mathrm{y}$ was unknown due to transfer to out-of-state facilities. Mean age at the time of discharge was 18.4 months and 16.7 months in the preTPVH and post-TPVH groups, respectively.

The Servo-i (Maquet, Rastatt, Germany) was the ICU ventilator utilized for all subjects in both groups. The PHV used by both groups was either the LTV 1200 (Vyaire 


\section{Transition From ICU Ventilator to Home Ventilator}

Table 4. Days From Initial Attempt to Successful Transition to PHV

\begin{tabular}{lcccc}
\hline \hline \multicolumn{1}{c}{ Group } & Pre-TPHV, $n$ & Days & Post-TPHV, $n$ & Days \\
\hline Overall & 27 & $67 \pm 91$ & 28 & $17 \pm 29$ \\
Neonatal ICU & 14 & $82 \pm 112$ & 17 & .01 \\
Other ICU & 13 & $50 \pm 60$ & 11 & .062 \\
Bronchopulmonary dysplasia & 10 & $39 \pm 29$ & 14 & $.13 \pm 24$ \\
\end{tabular}

Data are presented as mean $\pm \mathrm{SD}$

$\mathrm{PHV}=$ portable home ventilator

TPHV $=$ transition to portable home ventilator

Medical, Mettawa, Illinois) or the Trilogy 200 (Philips, Eindhoven, The Netherlands). The majority of all transition attempts occurred with the LTV 1200 , as $<5 \%$ of subjects from both groups were trialed using the Trilogy ventilator.

For children in the pre-TPHV group, there were a total of 112 attempts to transition to a PHV for 27 subjects. Of the total attempts, 23 were excluded due to acute illness ( $n=$ $21)$ or scheduled procedures $(n=2)$, leaving an adjusted total of 88 transition attempts. The exclusions following a scheduled procedure represented children who had already demonstrated stability with a PHV, and placement on the ICU ventilator post procedure was more of a precautionary measure. The mean number of total attempts was $3.3 \pm$ 2.7, and the mean number of failed attempts was $2.3 \pm 2.7$ in the pre-TPHV protocol group. In the post-TPHV protocol group, there were 54 total attempts with 28 children. Nine attempts were excluded for acute illness, yielding an adjusted total of 45 attempts for those who underwent the protocol. The mean number of total attempts was $1.6 \pm 1.1$ and $0.6 \pm 1.1$ failed attempts in the post-TPHV protocol group. There was a significant decrease in both the total number of transition attempts and the number of failed transition attempts for those who underwent the protocol $(P=.005)$.

The mean length of time from initial attempt with a PHV to successful transition was significantly reduced from $67 \pm 90.7 \mathrm{~d}$ to $17 \pm 28.6 \mathrm{~d}$ after initiation of the protocol $(P=.01)$. LOS was shortened from a mean of $13 \pm 6.4$ months before the protocol compared to $9 \pm 4.5$ months after the protocol was implemented $(P=.050)$. Overall, significance was not noted for age or weight at the time of the initial transition attempt.

\section{Subgroup Analyses}

Subjects in the Neonatal ICU. There were 31 subjects from the neonatal ICU, with 14 and 17 in the pre- and postTPHV groups, respectively. The majority of all subjects, ie, $68 \%(n=21,9$ pre-TPHV, 12 post-TPHV), had a diagnosis of BPD. The mean age at the time of the initial PHV attempt was $12 \pm 5.1$ months before the protocol and $11 \pm$ 3.3 months after the protocol was implemented $(P=.56)$.
There was a significant decrease in failed transition attempts for subjects in the neonatal ICU, from $3 \pm 3.1$ to $0.8 \pm 1.3$ attempts $(P=.02)$. The mean length of time to achieve successful transition was reduced from $82 \pm 112 \mathrm{~d}$ to $20 \pm 32 \mathrm{~d}$ and was approaching significance $(P=.062)$. The mean LOS in the neonatal ICU before transfer to the non-ICU ventilator unit was not significantly reduced as the pre-TPHV protocol group remained in the neonatal ICU for $289 \pm 131 \mathrm{~d}$ before transfer and $227 \pm 83 \mathrm{~d}$ in the postTPHV protocol group $(P=.14)$. Weight at the time of successful transition for subjects in the neonatal ICU was found to be significant $(P=.047)$. For those in the preTPHV protocol group who were in the neonatal ICU, mean weight was $9.5 \pm 2 \mathrm{~kg}$, whereas it was $8 \pm 2 \mathrm{~kg}$ when the protocol was utilized. Total LOS was shortened from $17 \pm$ 5 months to $12 \pm 3.1$ months for subjects in the neonatal $\operatorname{ICU}(P=.002)$.

Other ICU Subjects and Subjects With BPD. There were 24 children from other ICU locations, with 13 subjects in the pre-TPHV group and 11 in the post-TPHV group. Primary diagnoses for the need for ventilator support were highly variable and there was not a common theme. Children from other ICUs did not have a significant decrease in the mean number of failed attempts, with $1.5 \pm$ 2 and $0.4 \pm 0.7$ failures pre- and post-TPHV protocol, respectively $(P=.12)$. The mean number of days to achieve successful transition was reduced from $50 \pm 60 \mathrm{~d}$ to $13 \pm 24 \mathrm{~d}$ for subjects from other ICUs, but this was not statistically significant $(P=.09)$. No significant differences were found with any of the variables for the BPD subgroup. Table 4 summarizes the time required for successful transition overall for all subjects and by subgroups.

\section{Discussion}

Transition from an ICU ventilator to a PHV is an important step in the discharge planning process for children requiring long-term mechanical ventilation. Failure to tolerate the PHV could contribute to an increased LOS. Readiness for transition should also establish clinical stability in nonrespiratory systems, as other medical issues 


\section{Transition From ICU Ventilator to Home Ventilator}

could impact ability to tolerate a PHV. Children with complex secondary diagnoses such as omphalocele, congenital heart disease, or severe pulmonary hypertension may require more time following tracheostomy placement before they are stable enough to attempt the transition to a PHV. Additionally, sedation weaning may play a role in tolerating the transition away from the ICU ventilator. Many of the children in our study were weaning from narcotic sedation when the transition was attempted. It is not clear whether narcotic withdrawal symptoms or increased work of breathing related to ventilator intolerance contributed to failed attempts.

Ventilator nomenclature differs among manufacturers and is not standardized. ${ }^{22,23}$ Differences in how the ICU ventilator and PHV function may have been a contributing factor in unsuccessful trials. Bias flow is generally not adjustable or variable on some PHVs, which may result in increased work of breathing in smaller infants. ${ }^{15}$ In the subgroup analysis of subjects from other ICUs, a significant reduction in the number of failed attempts was not identified. This may be due in part to older children in the other ICU locations as compared to subjects in the neonatal ICU who were admitted at birth. However, there was a significant difference in weight at the time of successful transition for children from the neonatal ICU when the protocol was utilized. Weight was less in the post-TPHV protocol group, and we speculate that this may have been related to the decreased number of failed attempts and shortened time to achieve successful transition.

One subject from the pre-TPHV protocol group was excluded due to an atypical course with the PHV. This child had a history of BPD and a previous neonatal ICU hospitalization requiring mechanical ventilation, but the subject was able to wean off mechanical ventilation prior to discharge. Tracheostomy placement and long-term ventilation were required during a subsequent hospitalization in the pediatric ICU, followed by multiple unsuccessful trials with the PHV. Ultimately, due to rapid resolution of lung disease, this child was able to wean from ventilator support prior to discharge home.

Although LOS was reduced in subjects who were managed with the protocol, LOS may not be an accurate measure of change following interventions aimed at reduction of LOS because LOS can be influenced by both medical and nonmedical factors. While a child may have successfully transitioned to the PHV and demonstrated medical stability for discharge, other aspects of care may delay the process. Social factors can have a major impact on LOS and originate from several sources, such as the lack of a home capable of supporting ventilator equipment or caregiver ability to complete training. Reasons for delays in training range from transportation issues to challenges with demonstrating competency in skills. Lack of adequate staffing for home nursing, an ill-prepared home environment, and third-party payer issues can also result in delayed discharge. ${ }^{4,10-13}$ Postponed discharge for reasons of this nature increase the risk of hospital-acquired infections, which could further prolong hospitalization. There were 30 excluded transition attempts in our study due to acute illness rather than failure to tolerate the PHV.

Because there were several aspects of the readiness assessment and transition process, it is challenging to determine which feature may have had the greatest impact on reducing failed attempts and decreasing the length of time for successful transition. A predetermined transition plan with a standardized approach and readiness assessment as well as failure criteria, increased monitoring, and documentation were all key components of the protocol. Ventilator settings guidelines were also incorporated into the protocol.

The transition readiness assessment helped ensure that other factors in addition to the respiratory system were considered before a transition attempt, such as adequate growth, stable pulmonary hypertension, and status of sedation weaning, as these issues could influence a subject's ability to tolerate a PHV. We primarily used synchronized intermittent mandatory ventilation mode with pressure control (PC-SIMV) and pressure support, using the LTV 1200 ventilator in an attempt to standardize the transition process at our institution. Our transition process also took into consideration the secondary settings of the PHV, especially the trigger variable (ie, flow, time, pressure, or special algorithms), cycle variable (ie, flow, time, volume, or pressure), and limit variable. ${ }^{15,24}$ Adjustments to these variables in real time during the transition attempt can help improve synchrony with the ventilator and decrease the work of breathing, which ultimately results in a better-tolerated breathing pattern. ${ }^{15}$

Heightened monitoring during the first 48-72 h following the transition to a PHV and defining parameters for discontinuation of the trial may assist with preventing setbacks when the PHV trial is not tolerated. Discussing the cause of a failed transition attempt may help improve planning for the next attempt. These features of our protocol assisted with ensuring stability prior to attempting a transition to a PHV and provided a more objective means for outlining what constituted an unsuccessful attempt and return to the ICU ventilator.

There were several limitations to this quality-improvement study. This was a retrospective chart review that examined the process of transitioning to a PHV following implementation of a protocol. During the time encompassed by pre-TPHV protocol period (2011-2014) and the post-protocol period (2015-2018), other changes in care likely occurred that might have affected total length of hospital stay. This was unlikely due to the choice of ventilator or mode, as $>95 \%$ of transition attempts were using the LTV 1200 with PC-IMV and pressure support, both before and after implementation of the TPHV protocol. The mode 


\section{Transition From ICU Ventilator to Home Ventilator}

utilized was based on institutional practice and physician preference at the time the protocol was first introduced. The LTV 1200 utilizes an active patient circuit, whereas the Trilogy ventilator uses either an active or a passive circuit. We used only a passive circuit with the Trilogy ventilator, and our clinicians and providers had more experience with the LTV 1200 at that time. The LTV 1200 was the first choice for a PHV at the time of the study because the Trilogy ventilator was still relatively new to our practice and was not available to us until the middle of the preTPHV period. Very few PHV trials were performed using the Trilogy ventilator, and these data were not specifically captured. There were no subjects from the pre-TPHV group who successfully transitioned to the Trilogy ventilator, and only 1 subject in the post-TPHV protocol group successfully transitioned to the Trilogy ventilator using PC-IMV and pressure support.

Our assessment of readiness for transition to a PHV included clinical stability in a synchronized intermittent mandatory ventilation mode (Table 1). Near the end of the overall study period, newer portable ventilator models with volume-target ventilation modes became available. When these modes are used, it may be most appropriate to transition a patient from a volume-targeted mode on the ICU ventilator (eg, pressure-regulated volume control) to a volume-targeted mode on the PHV. Many newer models also have improved leak compensation and options for trigger, limit, and cycling variables to help improve patient-ventilator synchrony. Determination of whether and how this may affect the process of transition to a PHV will require future study.

The choice of PHV model should not only meet the needs of the patient but should also be one in which physicians, bedside clinicians, home care nurses, and respiratory therapists are knowledgeable and experienced. Although LOS in the neonatal ICU was not significantly reduced with use of the TPHV protocol, lower weight for subjects in the neonatal ICU at the time of successful transition may suggest that earlier transition to PHV could contribute to the shorter total LOS after TPHV protocol initiation.

A prospective design may have yielded additional data to provide more insight as to which portion of the protocol had the most impact on reducing failed attempts and length of time to successful transition. This was a single-center study that included only young subjects most of whom had BPD and most of whom were ventilated using one model of ventilator and predominately one mode of ventilation; therefore, these results may not apply to other age groups or patients with other indications for a PHV. A detailed review of ventilator settings utilized may have helped identify trends related to failed and successful attempts. Subject ethnicity was not evaluated, so it is unknown whether this aspect would reveal further information. Provider adherence to utilizing the protocol was not monitored, and therefore it is not clear if this had any influence on the failed transition attempts. Nonmedical factors that may have delayed discharge were not analyzed and would be challenging to extract from the medical record. Further research is needed to evaluate what impact sedation weaning may have on the process for changing from an ICU ventilator to a PHV.

\section{Conclusions}

In our experience, the use of a standardized process for transition to a PHV resulted in a significant reduction in both the number of failed attempts and the length of time to achieve successful transition. The implication for practice of earlier successful transition to a PHV may allow patients to be discharged sooner. Hospital LOS may be impacted by both medical and nonmedical factors, such as complicated medical conditions, response to sedation weaning, social issues, and availability of private duty nursing in the home setting. Future study is needed to further evaluate the process of successful transition to a PHV. A national home ventilator registry could assist with identifying trends and common factors associated with successful transition to a PHV. Additionally, clinical practice guidelines need to be developed to standardize the process for changing from an ICU ventilator to a PHV in children.

\section{REFERENCES}

1. McDougall CM, Adderley RJ, Wensley DF, Seear MD. Long-term ventilation in children: longitudinal trends and outcomes. Arch Dis Child 2013;98(9):660-665.

2. Murphy J. Medically stable children in PICU: better at home. Paediatr Nurs 2008;20(1):14-16.

3. Sahetya S, Allgood S, Gay PC, Lechtzin N. Long-term mechanical ventilation. Clin Chest Med 2016;37(4):753-763.

4. Sobotka SA, Foster C, Lynch E, Hird-McCorry L, Goodman DM. Attributable delay of discharge for children with long-term mechanical ventilation. J Pediatr 2019;212:166-171.

5. Fields AI, Rosenblatt A, Pollack MM, Kaufman J. Home care costeffectiveness for respiratory technology-dependent children. Am J Dis Child 1991;145(7):729-733.

6. King AC. Long-term home mechanical ventilation in the United States. Respir Care 2012;57(6):921-932.

7. Benneyworth BD, Gebremariam A, Clark SJ, Shanley TP, Davis MM. Inpatient health care utilization for children's dependent on long-term mechanical ventilation. Pediatrics 2011;127(6):e1553-e1541.

8. Overman AE, Liu M, Kurachek SC, Shreve MR, Maynard RC, Mammel MC, Moore BM. Tracheostomy for infants requiring prolonged mechanical ventilation: 10 years' experience. Pediatrics 2013;131(5):e1491-1496.

9. Amin R, Sayal A, Syed F, Daniels C, Hoffman A, Moraes TJ, Cox P. How long does it take to initiate a child on long-term invasive ventilation? Results from a Canadian pediatric home ventilation program. Can Respir J 2015;22(2):103-108.

10. Benscoter D, Borschuk A, Hart C, Voos K. Preparing families to care for ventilated infants at home. Semin Fetal Neonatal Med 2019;24 (5): 101042 . 


\section{Transition From ICU Ventilator to Home Ventilator}

11. Edwards EA, O’Toole M, Wallis C. Sending children home on tracheostomy dependent ventilation: pitfalls and outcomes. Arch Dis Child 2004;89(3):251-255.

12. Sobotka SA, Hird-McCorry LP, Goodman DM. Identification of fail points for discharging pediatric patients with new tracheostomy and ventilator. Hosp Pediatr 2016;6(9):552-557.

13. Maynard R, Christensen E, Cady R, Jacob A, Ouellette Y, Podgorski $\mathrm{H}$, et al. Home health care availability and discharge delays in children with medical complexity. Pediatrics 2019;143(1):e20181951.

14. Baker CD, Martin S, Thrasher J, Moore HM, Baker J, Abman SH, Gien J. A standardized discharge process decreases length of stay for ventilator-dependent children. Pediatrics 2016;137(4):e20150637.

15. Fierro JL, Panitch HB. Transitioning from an ICU ventilator to a portable home ventilator. Semin Fetal Neonatal Med 2019;24(5):101041.

16. O'Brien JE, Birnkrant DJ, Dumas HM, Haley SM, Burke SA, Graham RJ, Kharasch VS. Weaning children from mechanical ventilation in a post-acute care setting. Pediatr Rehabil 2006;9(4):365-372.

17. Sterni LM, Collaco JM, Baker CD, Carroll JL, Sharma GD, Brozek JL, et al. An official American Thoracic Society clinical practice guideline: pediatric chronic home invasive ventilation. Am J Respir Crit Care Med 2016;193(8):e16-e35.
18. Panitch HB, Downes JJ, Kennedy JS, Kolb SM, Parra MM, Peacock J, Thompson MC. Guidelines for home care of children with chronic respiratory insufficiency. Pediatr Pulmonol 1996;21(1):52-56.

19. Eigen H, Zander J. Home mechanical ventilation of pediatric patients. American Thoracic Society. Am Rev Respir Dis 1990;141 (1):258-259.

20. Jardine E, Wallis C. Core guidelines for the discharge home of the child on long term assisted ventilation in the United Kingdom. Thorax 1998;53(9):762-767.

21. Jardine E, O'Toole M, Paton JY, Wallis C. Current status of long term ventilation of children in the United Kingdom: questionnaire survey. BMJ 1999;318(7179):295-299.

22. Chatburn RL. Understanding mechanical ventilators. Expert Rev Respir Med 2010;4(6):809-819.

23. Mireles-Cabodevila E, Hatipoglu U, Chatburn RL. A rational framework for selecting modes of ventilation. Respir Care 2013;58(2):348366. Erratum in: Respir Care 2013;58(4):e51.

24. Chatburn RL, El-Khatib M, Mireles-Cabodevila E. A taxonomy for mechanical ventilation: 10 fundamental maxims. Respir Care 2014;59 (11):1747-1763.

This article is approved for Continuing Respiratory Care Education credit. For information and to obtain your CRCE

(free to AARC members) visit 